\title{
Frequency Modulation and Device Repaired by Laser Surface Treatment in Film Bulk Acoustic Filters
}

\author{
C.C. Cheng ${ }^{* 1}$, R.C. Lin $^{* 2}$, H.K. Lin $^{* 3}$, K.S. Kao ${ }^{* 4}$, and Y.C. Chen ${ }^{* 5}$ \\ ${ }^{* 1}$ Department of Electronic Engineering, De Lin Institute of Technology, Taipei, Taiwan \\ ${ }^{* 2}$ Electronics and Optoelectronics Research Laboratories, Industrial Technology Research Institute, Hsinchu, Taiwan \\ ${ }^{*}$ Graduate Institute of Materials Engineering, National Pingtung University of Science and Technology, Pingtung, Taiwan \\ E-mail:HKLin@mail.npust.edu.tw \\ ${ }^{* 4}$ Department of Computer and Communication, Shu-Te University, Kaohsiung, Taiwan \\ ${ }^{* 5}$ Department of Electrical Engineering, National Sun Yat-Sen University, Kaohsiung, Taiwan
}

\begin{abstract}
The study used a two-stage radio frequency magnetron sputtering method to deposit $\mathrm{ZnO}$ piezoelectric films. Lasers emitting 1064- and 355-nm wavelengths were used to conduct thermal treatment of the $\mathrm{ZnO}$ films. The measurement results of X-ray diffraction and scanning electron microscopy showed that ultraviolet lasers (UV) effectively improved the c-axis preferred orientation of $\mathrm{ZnO}$ films, facilitating the improvement of the return loss of longitudinal-mode film bulk acoustic resonator (FBAR) devices and their resonance frequency changes. Near-infrared lasers (NIR) enabled the growth of $\mathrm{ZnO}$ films along the a-axis, resulting in a deterioration of the return loss of longitudinal-mode FBAR devices. Finally, UV lasers were used to repair a FBAR filter that failed to modulate frequencies. The filter was modified into a band-pass filter after its frequency response was successfully modulated.
\end{abstract}

DOI: $10.2961 / \mathrm{jlmn} .2016 .01 .0002$

Keywords: Laser; film bulk acoustic resonator; $\mathrm{ZnO}$; Fiber

\section{Introduction}

Current acoustic devices used in radio frequency (RF) filters can be divided into two types, surface acoustic wave filters (SAWFs) [1-3] and film bulk acoustic resonator (FBAR) filters $[4,5]$, which have replaced piezoelectric films in conventional crystal and ceramic filters in bulk resonators to achieve high operating frequencies (exceeding gigahertz) [6]. Because frequency control is crucial to resonator devices, the frequencies of resonators connected in series and parallel directly influences filter bandwidths. In addition, frequency modulation influences the quality factor and insertion loss in these devices. Determining the optimal parameters between frequency modulation and other FBAR characteristics was the focus of this study.

Recently, with advancements in laser-pumping techniques, lasers have been increasingly applied in a growing number of fields, such as metal and ceramic cutting [7], welding [8], patterning [9, 10], and surface treatment [11-13]. The semiconductor and LED panel industries frequently use lasers as surface-treatment tools. Because lasers can be used to treat surfaces at selected areas and controlled depths, the film treatment process does not influence the characteristics of substrates and substantially assists in repairs of completed devices. The proposed FBAR filter requires resonators combined with two or more resonance frequencies on each substrate. Traditional resonator frequencies are modulated using the mass-loading effect $[14,15]$. Electrodes of differing thicknesses are typically used to modulate the resonance frequencies of serially connected resonators. However, this method requires an additional photomask and film deposition process, which is complex and time consuming. Thus, this study used laser-annealing techniques to control the crystallization of piezoelectric films and modulate the resonance frequencies of the resonators. Subsequently, after the device was completed, lasers were used to repair device defects, thereby reducing production costs and increasing yield rates.

\section{Experimental method}

This study used platinum for the bottom electrode of the FBAR and titanium for the seed layer; titanium and platinum were deposited using a dual-target DC sputtering system. Subsequently, a bottom electrode possessing low surface roughness and low sheet resistance were obtained [16]. In the FBAR device, the roughness and sheet resistance of the bottom electrode influenced the loss of RF signals [17] and the bottom electrode possessing low roughness substantially affected $\mathrm{ZnO}$ growth.

$\mathrm{ZnO}$ was deposited using a two-stage sputtering method at room temperature. During the first stage, a buffer layer possessing low surface roughness was deposited. During the second stage, sputtering parameters facilitating a high c-axis preferred orientation were used to produce $\mathrm{ZnO}$ piezoelectric films containing low surface roughness and high c-axis preferred orientation. The two-stage $\mathrm{ZnO}$ sputtering parameters are presented in our previous study [18]. The structure of an FBAR and an FBAR filter is shown in Fig. 1. An FBAR comprises a piezoelectric layer interlaid between metal films, forming a resonator, as 
shown in Fig. 1(a). An FBAR filter comprises a serial resonator and two parallel resonators, where the frequency responses of the serial and parallel connections join to form a band-pass filter, as shown in Fig. 1(b). The production process is presented as follows. A silicon substrate was washed according to the standard washing process stipulated by the Radio Corporation of America, and SiNx was grown on both sides using low-pressure chemical vapor deposition to generate a $\mathrm{KOH}$ etching mask for the silicon. Subsequently, a two-target DC sputtering system was used to grow a bottom electrode and $\mathrm{ZnO}$ piezoelectric films. $\mathrm{KOH}$ wet etching was used for back etching at a $30 \mathrm{wt} \%$ $\mathrm{KOH}$ concentration and etching temperature at $100{ }^{\circ} \mathrm{C}$. Consequently, the FBAR and FBAR filters were constructed.

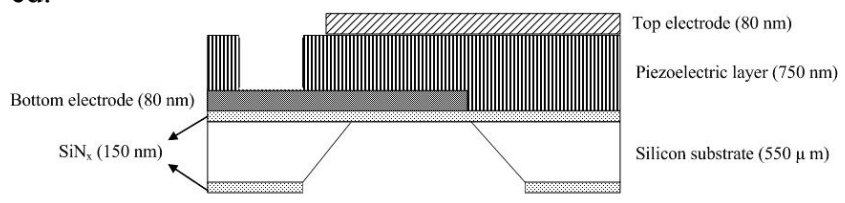

(a)

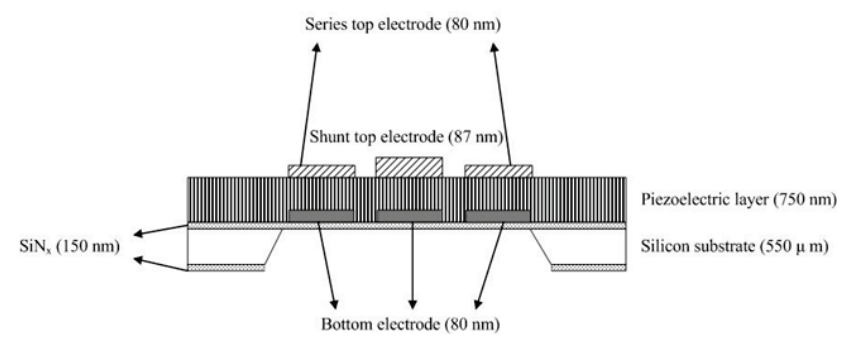

(b)

Fig. 1 Device structures of (a) FBAR and (b) FBAR filter.

Regarding the laser treatment, the laser experiments were conducted using a UV laser (Coherent AVIA 3557000 ) with a wavelength of $355 \mathrm{~nm}$, a repetition rate of 40 $\mathrm{kHz}$, and a pulse duration of $<35 \mathrm{~ns}$, and an Near-infrared laser (SPI G3) with a wavelength of $1064 \mathrm{~nm}$, repetition rate of $25 \mathrm{kHz}$, and pulse duration of $>10 \mathrm{~ns}$. The laser treatment for UV and NIR condition was carried out at a laser power of $759 \mathrm{~mW}$ and $700 \mathrm{~mW}$, respectively. The treatment process employed defocused laser scanning at a scanning rate of $2 \mathrm{~mm} / \mathrm{s}$.

In order to increase the resolution of the pattern, the size of the laser spot was decreased. The diameter of the laser spot, D0, is expressed as:

$$
D_{0}=1.22 \times\left(\frac{\lambda \times F}{n \times W_{d}}\right) \times M^{2}
$$

where $\lambda$ is the laser wavelength, $F$ is the focal length, $\mathrm{n}$ is a refractive index, $\mathrm{Wd}$ is the diameter of the incident laser, and M2 is the laser-quality factor. Equation (1) indicates that the diameter of the laser beam is directly proportional to the wavelength and focal length. For the experiment, the spot size was $40 \mu \mathrm{m}$ for the laser system.

Regarding the analysis of physical properties, field emission scanning electron microscopy (Philips XL40 FESEM) was used to observe the surface morphology and cross-sectional structure of each film layer. X-ray diffraction (XRD) was used to analyze the crystal characteristics of the piezoelectric film. The frequency response of the FBAR filters was measured using HP8720 network analyzer and CASCADE high-frequency probe (RHM-06/V + GSG 150).

\section{Results and discussions}

The quality of $\mathrm{ZnO}$ piezoelectric films is the crucial factor that influences the quality of FBAR devices. Numerous studies have shown that $\mathrm{ZnO}$ films with a superior caxis (002) preferred orientation present favorable piezoelectric characteristics in longitudinal waves [9-11]. Therefore, in this study, $\mathrm{ZnO}$ films were treated using differing laser parameters.

Fig. 2(a) shows the XRD result of the $\mathrm{ZnO}$ films treated using UV lasers, indicating that the treated $\mathrm{ZnO}$ films exhibited strong preference to the c-axis orientation. Fig. 2(b) shows that $\mathrm{ZnO}$ films demonstrated recrystallization after being treated by high-intensity lasers. The band gap of $\mathrm{ZnO}$ is approximately $3.4 \mathrm{eV}$, matching the $\mathrm{UV}$ absorption wavelength (3.49 eV for $355 \mathrm{~nm}$ ). Thus, $\mathrm{ZnO}$ possesses superior UV laser absorbance, resulting in clearly improved crystal characteristics of films after exposure to UV radiation.

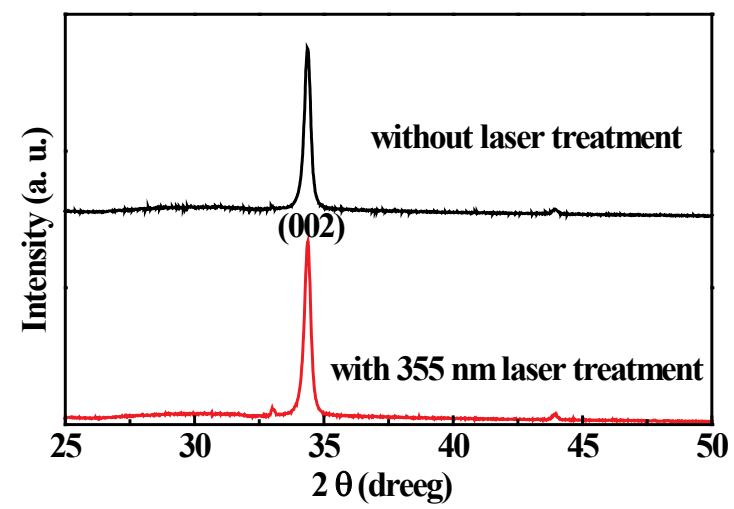

(a)

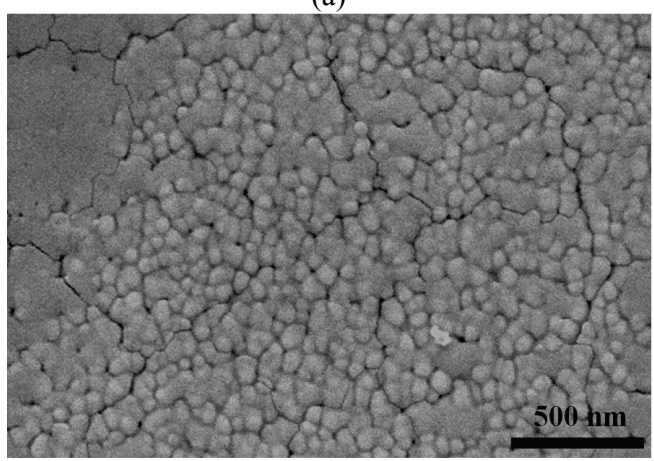

(b)

Fig. 2 (a) XRD result with and without $355 \mathrm{~nm}$ laser treatment and (b) SEM image of $\mathrm{ZnO}$ thin film with $355 \mathrm{~nm}$ laser treatment.

Fig. 3(a) shows the XRD result of the $\mathrm{ZnO}$ films treated using NIR lasers. After NIR laser treatment, a-axis (100) crystal peak grew and inhibited the c-axis (002) preferred orientation. The SEM result of Fig. 3(b) shows that $\mathrm{ZnO}$ films exhibit nanoparticles and a rough surface. The surface became roughened because NIR lasers treated the $\mathrm{ZnO}$ films using thermal energy, which led to phase transition in the $\mathrm{ZnO}$ films and the evaporation of partial $\mathrm{ZnO}$ films. 


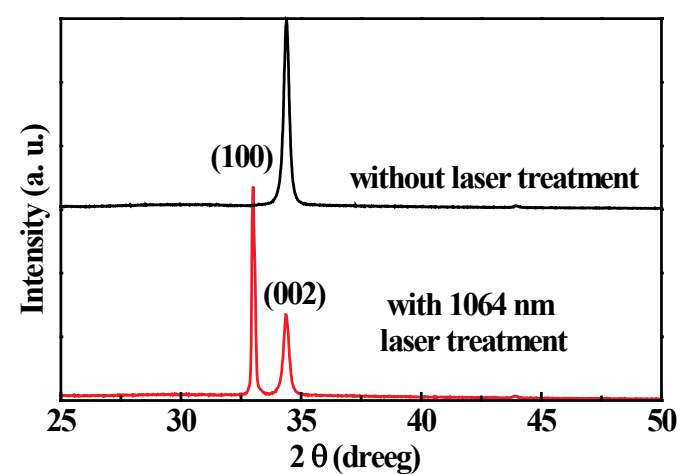

(a)

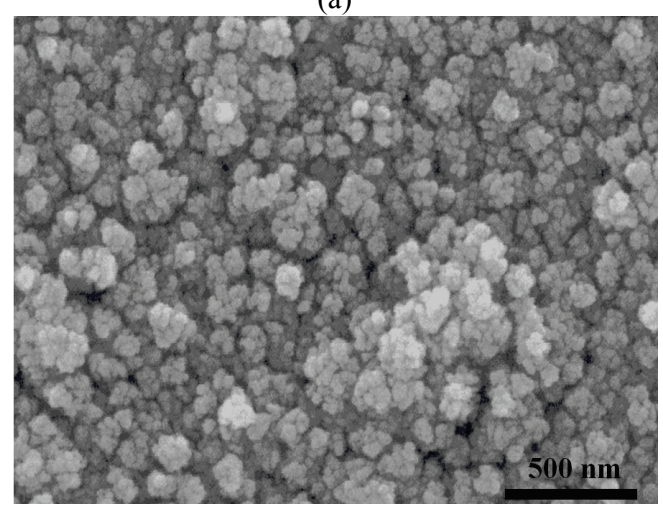

(b)

Fig. 3 (a) XRD result with and without $1064 \mathrm{~nm}$ laser treatment and (b) SEM image of $\mathrm{ZnO}$ thin film with $1064 \mathrm{~nm}$ laser treatment.

The frequency response of FBAR devices is shown in Fig. 4(a). FBAR devices that were produced using the $\mathrm{ZnO}$ films treated by UV lasers demonstrated a substantially increased return loss of frequency response because of the $\mathrm{ZnO} \mathrm{c}$-axis preferred orientation; the resonance frequency increased from $2472 \mathrm{MHz}$ to $2517 \mathrm{MHz}$. Fig. 4(b) shows the FBAR device fabricated using the $\mathrm{ZnO}$ films treated by NIR lasers. The density of the annealed thin film became dense so the frequency was shifted to the high frequency range. The return loss of the NIR laser-treated device decreased because the original $\mathrm{ZnO}$ c-axis preferred orientation was changed to a-axis preferred orientation. Therefore, the piezoelectric characteristics of longitudinal waves deteriorated, resulting in reduced frequency response of the device.

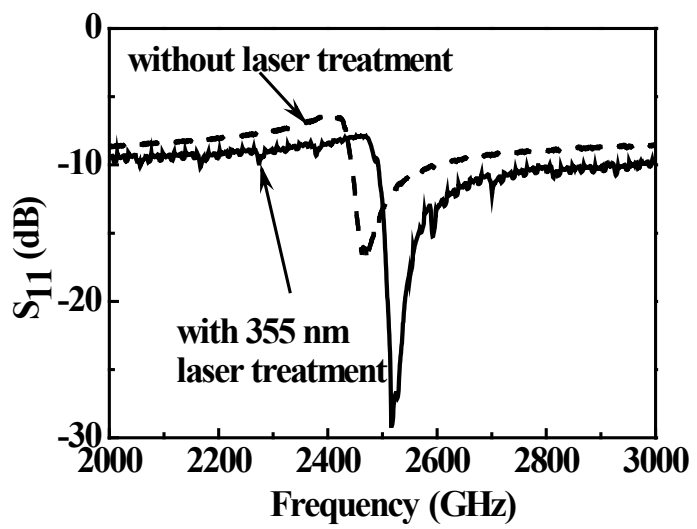

(a)

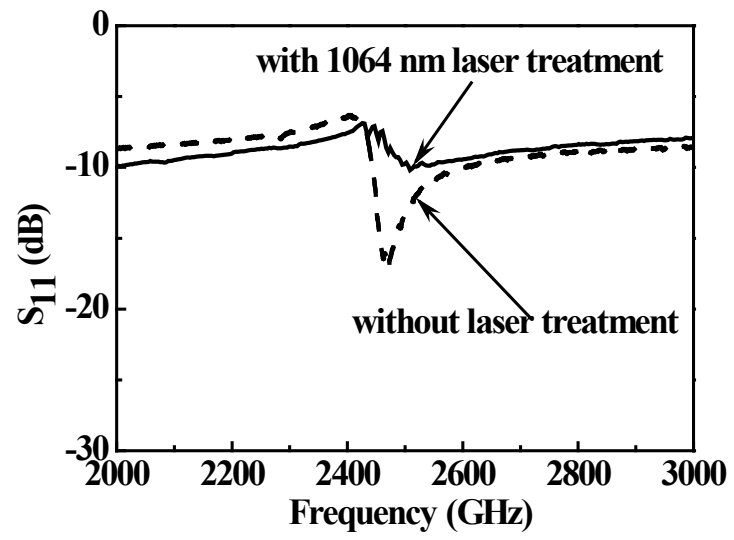

(b)

Fig. 4 (a) Frequency response of FBAR with and without $355 \mathrm{~nm}$ laser treatment, (b) Frequency response of FBAR with and without $1064 \mathrm{~nm}$ laser treatment.

Based on the laser repair results, UV lasers can regrow $\mathrm{ZnO} \mathrm{c}$-axis and improve the return loss and resonance frequency of frequency response. Therefore, the FBAR filter that failed to modulate frequencies in this study was selected to conduct laser repair. Fig. 5 indicates the frequency response of the FBAR filters, where black lines represent the filter that failed to modulate frequencies, exhibiting two central frequencies formed by the mismatched serial and parallel resonance frequencies. The white lines in Fig. 5 represent the frequency response repaired by UV lasers. After exposure to UV lasers, the resonance frequency and return loss of the $\mathrm{ZnO}$ c-axis were improved, increasing the resonance frequency of the serial resonator and inhibiting the frequency response of the parallel resonators. This result indicated that the FBAR filter was successfully repaired.

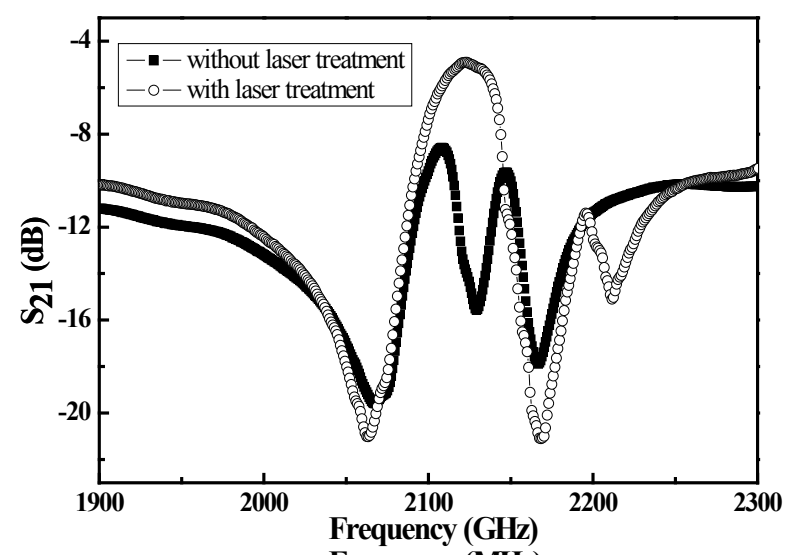

Fig. 5 Frequency response of FBAR filter with and without 355 nm laser treatment.

\section{Conclusions}

This study successfully constructed a FBAR device and FBAR filters and repaired an FBAR filter using an innovative laser treatment method. The results indicated that differing laser wavelengths influenced the crystal structure and surface morphology of $\mathrm{ZnO}$ films. UV lasers can re-grow the c-axis of $\mathrm{ZnO}$ and change the resonance frequencies of FBAR as a frequency modulator. In addition, this study successfully applied UV lasers to repair an FBAR filter. Moreover, although NIR lasers cannot effec- 
tively improve the c-axis preferred orientation of $\mathrm{ZnO}$, NIR lasers cause considerable growth along the a-axis. This result can be used as a reference for studies investigating $\mathrm{ZnO}$ films growing along different axes, providing high research value and applicability.

\section{References}

[1] K.C. Colin, in: Proceedings of the IEEE, 1989, pp. $1453-1484$

[2] K. Lange, B.E. Rapp, M. Rapp, Analytical and bioanalytical chemistry, 391, (2008) 1509-1519.

[3] A. Afzal, N. Iqbal, A. Mujahid, R. Schirhagl, Analytica chimica acta, 787, (2013) 36-49.

[4] R. Serhane, S. Abdelli-Messaci, S. Lafane, H. Khales, W. Aouimeur, A. Hassein-Bey, T. Boutkedjirt, Applied Surface Science, 288, (2014) 572-578.

[5] X. Zhang, W.C. Xu, J. Chae, Sensor Actuat a-Phys, 166, (2011) 264-268.

[6] M. Åberg, M. Ylimaula, M. Ylilammi, T. Pensala, A. Rantala, Analog Integrated Circuits and Signal Processing, 50, (2007) 29-37.

[7] H.-K. Lin, C.-J. Lee, T.-T. Hu, C.-H. Li, J.C. Huang, Optics and Lasers in Engineering, 50, (2012) 883-886.

[8] E. Akman, A. Demir, T. Canel, T. Sinmazçelik, Journal of Materials Processing Technology, 209, (2009) 37053713.

[9] H.K. Lin, C.H. Li, S.H. Liu, Optics and Lasers in Engineering, 48, (2010) 1008-1011.

[10] H.K. Lin, R.C. Lin, C.H. Li, Thin Solid Films, 518, (2010) 7253-7257.

[11] S.M.d. Nicolás, D. Muñoz, C. Denis, J.F. Lerat, T. Emeraud, Energy Procedia, 27, (2012) 586-591.

[12] C.J. Lee, H.K. Lin, C.H. Li, L.X. Chen, C.C. Lee, C.W. Wu, J.C. Huang, Thin Solid Films, 522, (2012) 330-335.

[13] P. Harcuba, L. Bacakova, J. Strasky, M. Bacakova, K. Novotna, M. Janecek, Journal of the mechanical behavior of biomedical materials, 7, (2012) 96-105.

[14] M. Hara, M. Ueda, Y. Satoh, Ultrasonics, 53, (2013) 90-96.

[15] R.C. Lin, Y.C. Chen, W.T. Chang, C.C. Cheng, K.S. Kao, Sensors and Actuators A: Physical, 147, (2008) 425429.

[16] R.C. Lin, K.S. Kao, C.C. Cheng, Y.C. Chen, Thin Solid Films, 516, (2008) 5262-5265.

[17] K.W. Tay, C.L. Huang, L. Wu, M.S. Lin, Japanese Journal of Applied Physics, 43, (2004) 5510.

[18] R.C. Lin, Y.C. Chen, K.S. Kao, Applied Physics A, 89, (2007) 475-479.

(Received: May 21, 2015, Accepted: January 4, 2016)

\section{Acknowledgments}

The authors gratefully acknowledge the financial support provided to this study by the National Science Council of Taiwan under Project No. NSC102-2221-E-020-009 and MOST 103-2221-E-020-010. 